\title{
Results from ZEUS at HERA
}

\author{
Bernd Loehr* \\ DESY \\ On behalf of the ZEUS collaboration \\ E-mail: bernd. loehredesy.de
}

New results are reported from the ZEUS experiment at HERA on deep ineleastic scattering and photoproduction in electron/positron proton collisions. These are mainly results on charm production cross-sections but include also results of a combination of older charm measurements from $\mathrm{H} 1$ and ZEUS and charm fragmentation functions. Also reported are results on inelastic $J / \Psi$ and $\Psi^{\prime}$ photoproduction and on photoproduction of prompt photons together with a jet.

The XXI International Workshop High Energy Physics and Quantum Field Theory

June 23 ? June 30, 2013

Saint Petersburg Area, Russia

${ }^{*}$ Speaker. 


\section{Introduction}

In HERA, electrons or positrons with energy of $E_{e}=27.5 \mathrm{GeV}$ collide with protons of $E_{p}=$ $920 \mathrm{GeV}$ resulting in a center of mass energy, $\sqrt{s}=318 \mathrm{GeV}$. In what follows the word electrons is used generically and includes also positrons where not specifically distiguished between them. HERA operated from 1992 to 2000 in a first running period, called HERAI, and has then been upgraded to higher instantaneous luminosity in the years 2000 to 2002. The HERAII running period ended in July 2007. The integrated luminosity for HERAI is about $120 \mathrm{pb}^{-1}$ and for HERAII about $380 \mathrm{pb}^{-1}$. Towards the end of the HERAII running period, data have been taken with lower proton energies with an integrated luminosity of about $65 \mathrm{pb}^{-1}$.

The ZEUS detector at HERA is a hermetic multipurpose detector consisting, from the inside out, of a tracking system, a solenoidal superconducting coil, and a uranium-scintillator sampling calorimeter. An iron yoke surrounds the calorimter. It is instrumented with muon chambers in front and behind the iron and proportional chambers inside the yoke which is made from iron slabs. A dedicated muon spectrometer is placed behind the iron yoke in the direction of the proton beam. During the shutdown from 2000 to 2002, the ZEUS detector has been equipped with a silicon strip micro vertex-detector (MVD). The higher integrated luminosity from the HERAII running allowed to improve the precision of results from HERAI and made possible new analyses. The MVD enabled the detection of secondary vertices and opened a new method to study heavy quark production using the measured decay lenghts of charm and beauty particles. After the end of the HERA running, one of the major tasks left is to combine results from the H1 and the ZEUS experiments to common HERA results.

\section{Combination of $\mathbf{H 1}$ and ZEUS results on charm production}

Charm production in DIS at HERA occurs in leading order through boson-glun fusion. The corresponding diagram for the process at HERA is shown in figure 1.

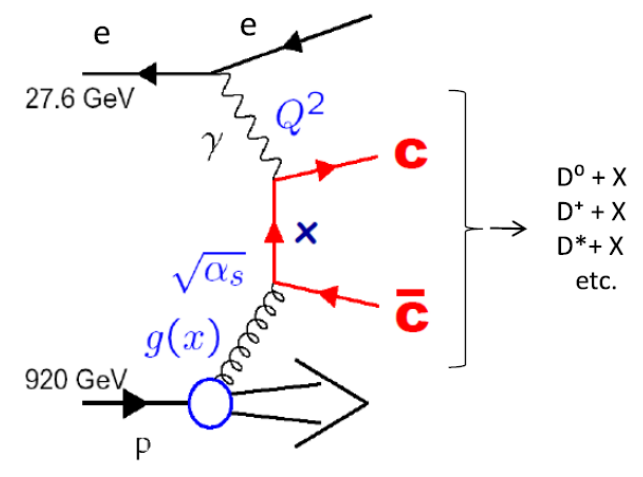

Figure 1: Charm production through bosongluon fusion at HERA.
The kinematic of the reaction is described by:

$$
\begin{aligned}
& s=(p+k)^{2} ; \quad Q^{2}=-q^{2}=\left(k-k^{\prime}\right)^{2} \\
& x=\frac{q^{2}}{2 p \cdot q} ; y=\frac{p \cdot q}{p \cdot k} ; z^{D}=\frac{p \cdot p^{D}}{p \cdot q}
\end{aligned}
$$

The ep center of mass energy squared is $s$, $k$ and $k^{\prime}$ are the four momenta of incoming and outgoing electrons, $\mathrm{p}$ is the four momentum of the incoming proton. $x$ is the Björken variable, $y$ is the inelasticity, and $z^{D}$ is the fraction of the virtual photon energy transfered to the charmed meson. 
Charm production contributes up to $30 \%$ of the total cross-section. It is a very good testing ground for perturbative QCD: it tests directly the gluon content of the proton, it involves multiple hard scales, $\mathrm{Q}^{2}, \mathrm{~m}_{\text {charm }}$ and $\mathrm{p}_{\mathrm{T}}$ of the produced charm particles. In addition it implies the problem of a perturbative procedure when $\mathrm{Q}^{2}$ or another hard scale crosses the threshold for charm quark production.

The double differential charm cross-section is given by two structure functions:

$$
\frac{d^{2} \sigma^{c \bar{c}}}{d x d Q^{2}}=\frac{2 \pi \alpha_{e m}^{2}}{x Q^{4}}\left(\left[1+(1-y)^{2}\right] F_{2}^{c \bar{c}}\left(x, Q^{2}\right)-y^{2} F_{L}^{c \bar{c}}\left(x, Q^{2}\right)\right) .
$$

Often, the reduced charm cross-section is used:

$$
\sigma_{\text {red }}^{c \bar{c}}=\frac{d^{2} \sigma^{c \bar{c}}}{d x d Q^{2}} \cdot \frac{x Q^{4}}{2 \pi \alpha_{e m}^{2}\left(1+(1-y)^{2}\right)}=F_{2}^{c \bar{c}}-\frac{y^{2}}{1+(1-y)^{2}} F_{L}^{c \bar{c}} .
$$

The H1 and ZEUS measurements of $\sigma_{\text {red }}^{c \bar{c}}$ have been projected onto a common $\left(x, Q^{2}\right)$ grid and have then been combined [1]. For systematic errors the correlations have been taken into account. Figure 2 shows the combined data, labeled HERA, as functions of $x$ for different $Q^{2}$ bins from $2.5 \mathrm{GeV}^{2}$ to $2000 \mathrm{GeV}^{2}$ together with HERAPDF1.5 NLO predictions [2] which have been derived from inclusive DIS data. To demonstrate the gain in precision by the combination the original measurements and the combined results are displayed in figure 3 for $Q^{2}=18 \mathrm{GeV}^{2}$.

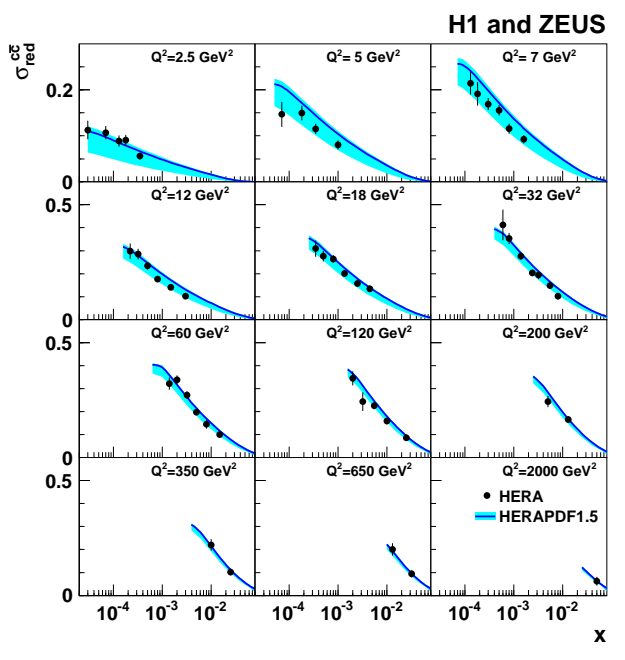

Figure 2: Combined H1 and ZEUS results for $\sigma_{\text {red }}^{c \bar{c}}$ and comparison to HERAPDF1.5 NLO predictions.

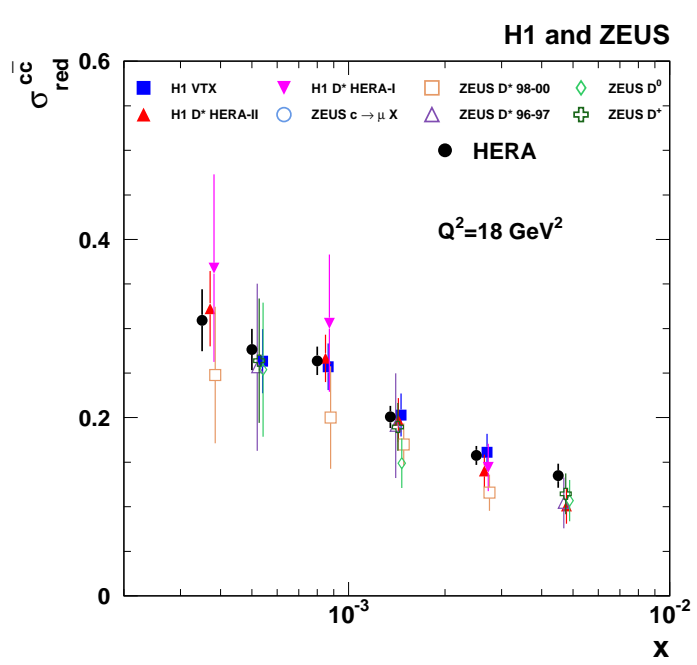

Figure 3: Individual measurements of $\sigma_{\text {red }}^{c \bar{c}}$ by $\mathrm{H} 1$ and ZEUS in comparison with the combined result for $Q^{2}=18 \mathrm{GeV}^{2}$.

In pQCD fits, like the fitting of PDFs, the question comes up how to treat the crossing of the threshold for the production of charmed particles. For HERAPDF1.5 NLO the RT standard scheme [3] with $m_{c}=1.4 \mathrm{GeV}^{2}$ has been used. The error band of the HERAPDF1.5 predictions are dominated 
by the variation $1.35 \leq m_{c} \leq 1.65 \mathrm{GeV}^{2}$.

Various heavy quark mass schemes exist:

- Fixed flavour number scheme (FFNS): only light quarks exist in the proton, heavy quarks are massive and are produced only in the hard process;

- Zero mass variable flavour number scheme (ZM-VFNS): below the threshold at $Q^{2} \approx m_{c}^{2}$ the charm cross section is zero and only three active flavours exist in the proton, above the threshold, charm is a massless active parton;

- General mass variable flavour number scheme (GM-VFNS): at low $Q^{2}$ it is like FFNS, at high $Q^{2}$ like ZM-VFNS, at intermediate scales several interpolation procedures exist.

The HERAI inclusive and charm data have been used to perform pQCD fits in various heavy flavour schemes with the charm mass as a free parameter and to determine the optimal charm mass for each scheme. Figure 4 shows the $\chi^{2}$ distributions and the positions of the optimal values $m_{c}^{o p t}$ for the schemes: RT standard, RT optimised [4], ACOT-full and S-ACOT- $\chi$ [5], and ZM-VFNS [6].

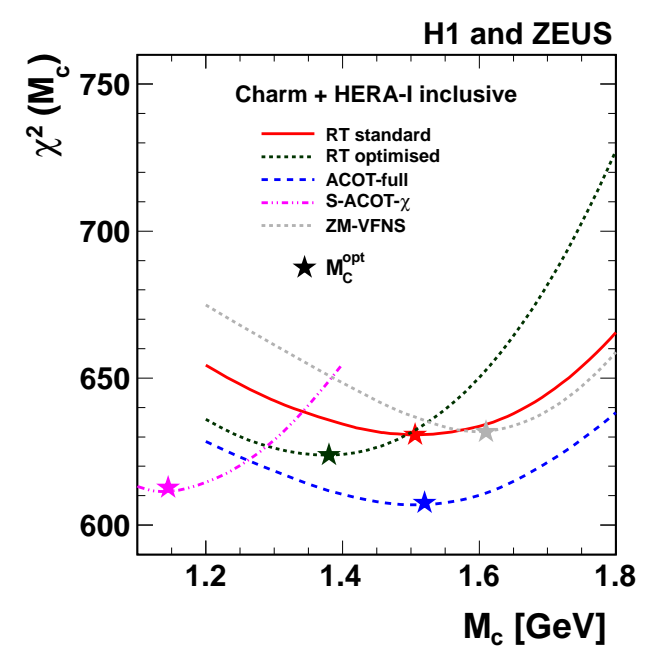

Figure 4: Determination of optimal charm masses for various heavy flavour schemes.

The use of the respective optimal charm mass in each heavy flavour scheme reduces the differences between predictions from the various schemes considerably. This is demonstrated for predictions of $\sigma_{W^{+}}, \sigma_{W^{-}}$, and $\sigma_{Z}$ at LHC for $\sqrt{s}=7 \mathrm{TeV}$ in figures 5-7. If the optimal charm masses are used the predictions vary by $2 \%$ for $\sigma_{W^{+}}$, by $1.4 \%$ for $\sigma_{W^{-}}$, and by $1.8 \%$ for $\sigma_{Z}$. 


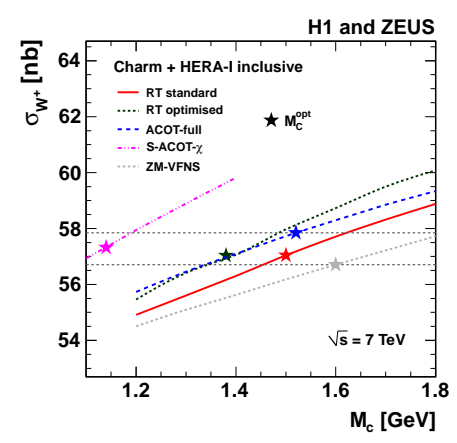

Figure 5: Predictions for $\sigma_{W^{+}}$at LHC from various heavy flavour schemes.

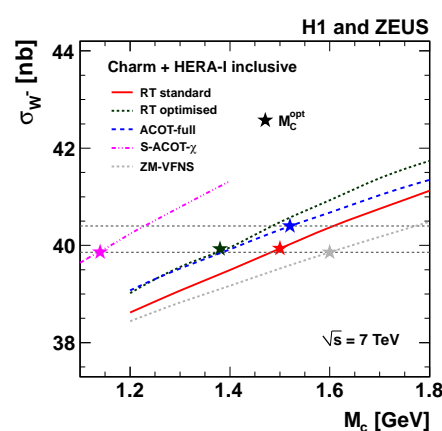

Figure 6: Predictions for $\sigma_{W^{-}}$at LHC from various heavy flavour schemes.

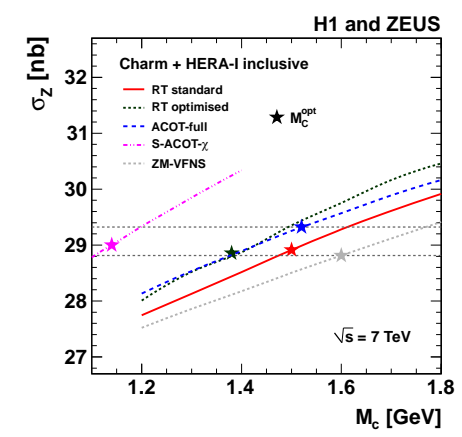

Figure 7: Predictions for $\sigma_{Z}$ at LHC from various heavy flavour schemes.

\section{3. $\mathrm{D}^{ \pm}$and $\mathrm{D}^{*}$ production from the HERAII data}

During the HERAII running period the ZEUS collaboration operated a micro-vertex detector. This was used to identify D mesons and to determine differential $D^{ \pm}$cross sections [7] in the kinematic region:

$$
\begin{gathered}
5 \leq Q^{2} \leq 1000 \mathrm{GeV}^{2}, \quad 0.02 \leq y \leq 0.7 \\
1.5 \leq p_{T}\left(D^{ \pm}\right) \leq 15 \mathrm{GeV}^{2}, \quad\left|\eta\left(D^{ \pm}\right)\right| \leq 1.6 .
\end{gathered}
$$

The $\mathrm{D}^{ \pm}$mesons were identified through their decay

$$
\mathrm{D}^{ \pm} \rightarrow \mathrm{K}^{\mp} \pi^{ \pm} \pi^{ \pm}
$$

and selected with the micro-vertex detector by cuts on the measured decay length. Figure 8 shows the $\mathrm{D}^{+}$signal.

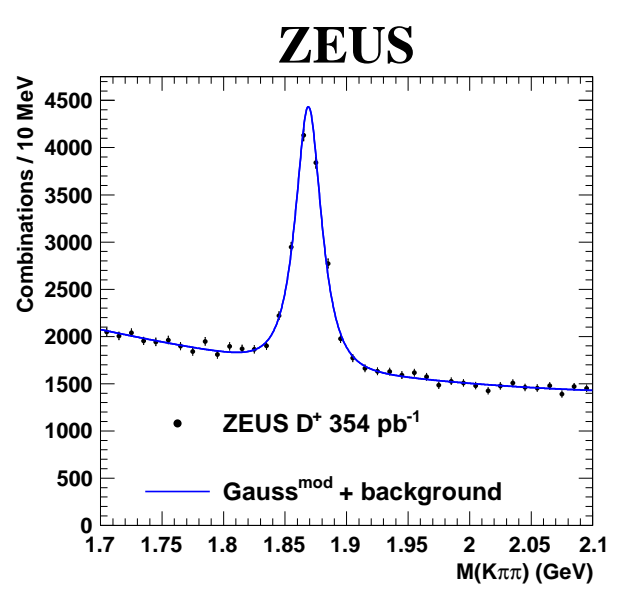

Figure 8: $\mathrm{D}^{+}$signal measured by ZEUS using cuts on the decay length measured with the micro-vertex detector together with a fit to the signal plus background.

Due to the higher integrated luminosity compared to the HERAI data single and double differential cross-sections can be determined. The single differential cross-section $d \sigma / d Q^{2}$ is shown in figure 9. The data are also compared to HERAI measurements with an integrated luminosity of $133.6 p b^{-1}$.

Figures 10 presents $d \sigma / d y$ for $D^{+}$production as a function of $y$ for different $Q^{2}$ bins. The data are compared to NLO calculations with the HVQDIS program [8]. Given the uncertainties of the NLO calculations there is good agreement between the data and the calculations. 


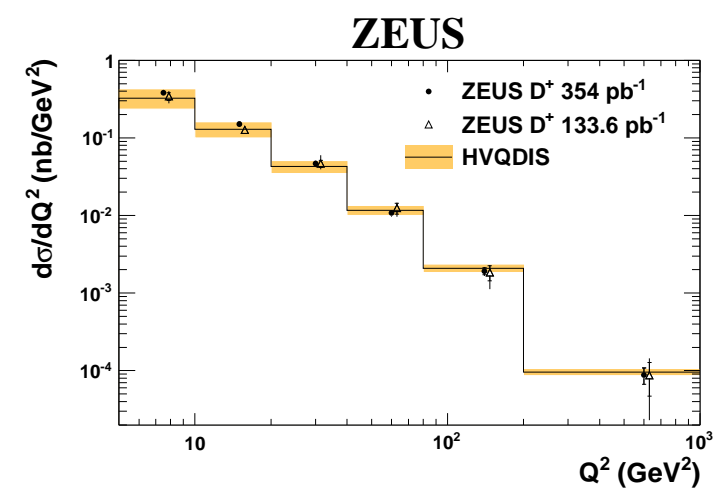

Figure 9: $d \sigma / d Q^{2}$ as a function of $Q^{2}$. The HERAII data are compared to the HERAI data and to a HVQDIS NLO calculation.
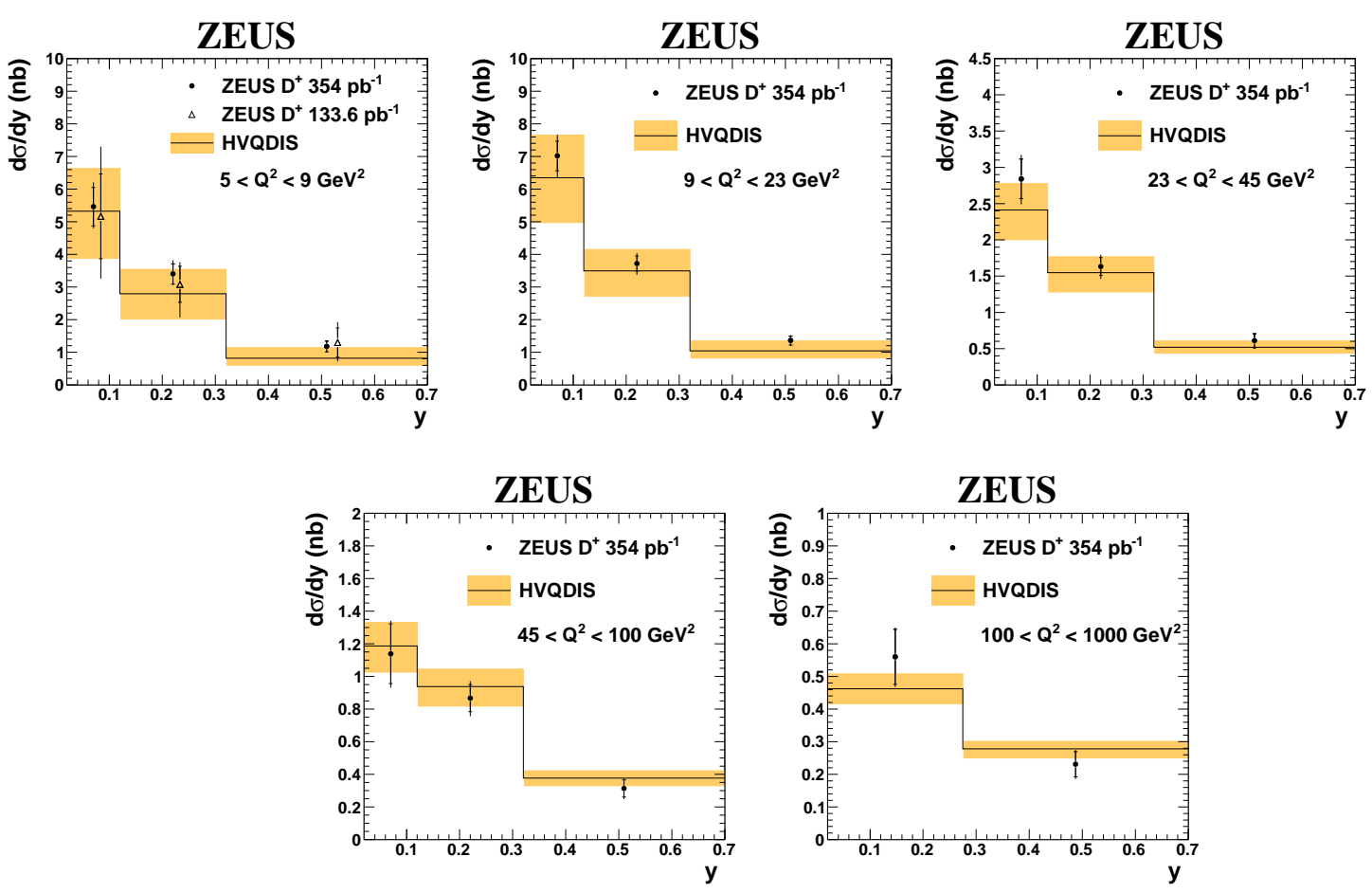

Figure 10: Double differential cross-sections, $d \sigma / d y$, as a function of $y$ for different bins in $Q^{2}$ are presented. The data are compared to HVQDIS NLO calculations. For the bin $5 \leq Q^{2} \leq 9 \mathrm{GeV}^{2}$ the HERAII data are shown together with the older HERAI data.

Cross-sections for $D^{*}$ production have been extracted from the HERAII data [9] using the reaction $e p \rightarrow e^{\prime} D^{* \pm} X$ and $D^{*+} \rightarrow D^{0} \pi_{s}^{+}$followed by $D^{0} \rightarrow K^{-} \pi^{+}$including charge conjugate states. The kinematic region of the extracted cross-sections is $5 \leq Q^{2} \leq 1000 \mathrm{GeV}^{2}, 0.02 \leq y \leq 0.7$ and $1.5 \leq \mathrm{p}_{\mathrm{T}}^{\mathrm{D}^{*}} \leq 20 \mathrm{GeV}, \quad\left|\eta^{\mathrm{D}^{*}}\right| \leq 1.5$. Figure 11 and figure 12 show the observed $D^{0}$ and $D^{*}$ signals.

The number of events from the HERAII data is sufficient to determine double differential cross- 


\section{ZEUS}

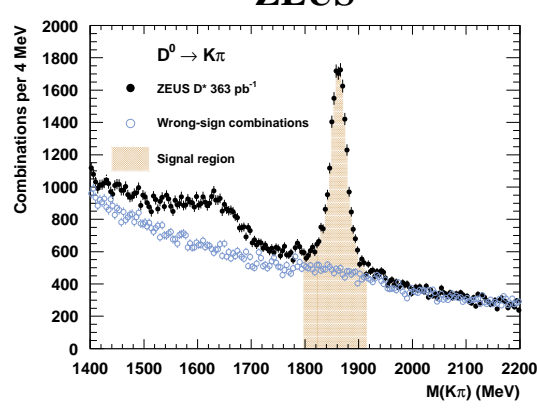

Figure 11: $D^{0}$ signal from $D^{0} \rightarrow K \pi$. The background in the signal region is estimated from the wrong sign combinations of $K \pi$ pairs.

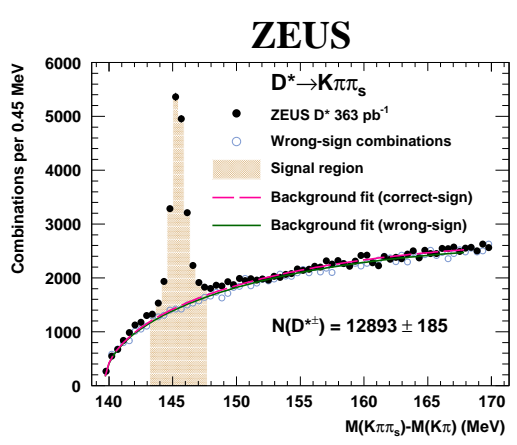

Figure 12: The $D^{*}$ signal from $D^{*} \rightarrow K \pi \pi_{s}$ after selecting $D^{0}$ candidates first. The background is estimated from a fit and also from wrong charge combinations.

sections for inclusive $D^{*}$ production in the quoted kinematical region. In figure $13, d^{2} \sigma / d y d Q^{2}$ is shown as a function of $y$ for different $Q^{2}$ bins. The ZEUS results are compared to H1 results and to HVQDIS plus Monte Carlo model calculations. There is fair agreement between the two experiments and also with the NLO calculation from HVQDIS.

\section{ZEUS}
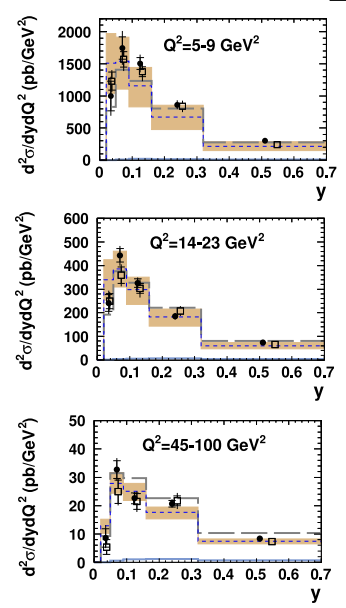
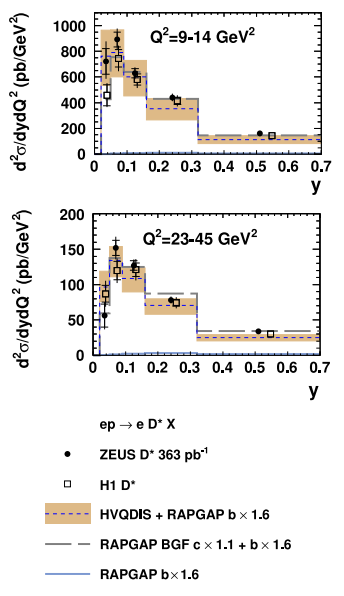

ZEUS
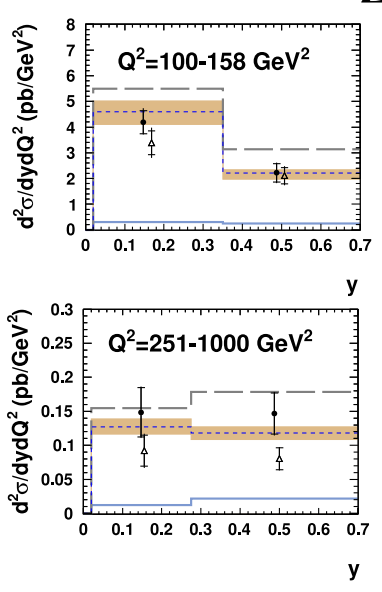

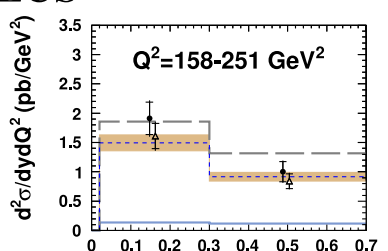

y

ep $\rightarrow$ e D* X

- ZEUS $D^{\star} 363 \mathrm{pb}^{-1}$

$\triangle \quad H 1 D^{*}\left(\right.$ high $\left.Q^{2}\right)$

HVQDIS + RAPGAP $b \times 1.6$

RAPGAP BGF $c \times 1.1+b \times 1.6$

RAPGAP $b \times 1.6$

Figure 13: $d^{2} \sigma / d y d Q^{2}$ for $D^{*}$ production from ZEUS and $\mathrm{H} 1$ as a function of $y$. The data are compared to HVQDIS NLO plus RAPGAP model calculations.

The cross-section data for $D^{+}$and $D^{*}$ production have been used to calculate the inclusice charmanticharm reduced cross-section $\sigma^{c \bar{c}}$ according to

$$
\frac{d^{2} \sigma^{c \bar{c}}}{d x d Q^{2}}=\frac{2 \pi \alpha_{e m}^{2}}{x Q^{4}}\left(1+(1-y)^{2}\right) \sigma_{r e d}^{c \bar{c}}\left(x, Q^{2}\right)
$$

Figure 14 shows $\sigma^{c \bar{c}}$ as a function of $x$ for different $Q^{2}$ bins as determined from the inclusive $D^{+}$ data and the inclusive $D^{*}$ data. These cross-sections are compared to the ones from the combined 
HERA $D^{+}$data (see above). Note that the ZEUS data presented here are not yet included in the HERA combination of $D^{+}$data. There is good agreement between all three datasets. Figure 15 shows $\sigma^{c \bar{c}}$ from the ZEUS $D^{*}$ data compared to the prediction from HERAPDF1.5 NLO with $m_{c}=1.5 \mathrm{GeV}$. Within the uncertainties of the data and of the HERAPDF1.5 predictions, indicated by the error band, there is good agreement.

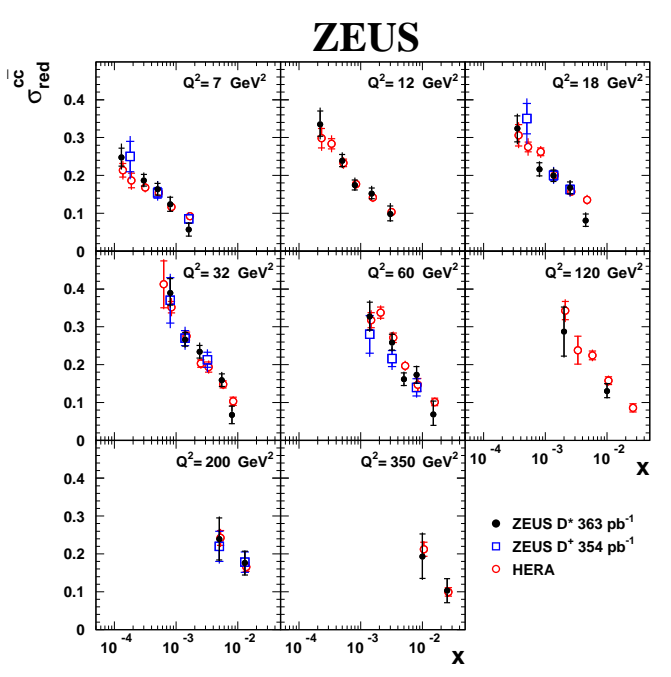

Figure 14: $\sigma^{c \bar{c}}$ determined from the inclusive $D^{ \pm}$data and from the inclusive $D^{*}$ data. These cross-sections are compared to $\sigma^{c \bar{c}}$ as derived from the H1-ZEUS combination of charm data.

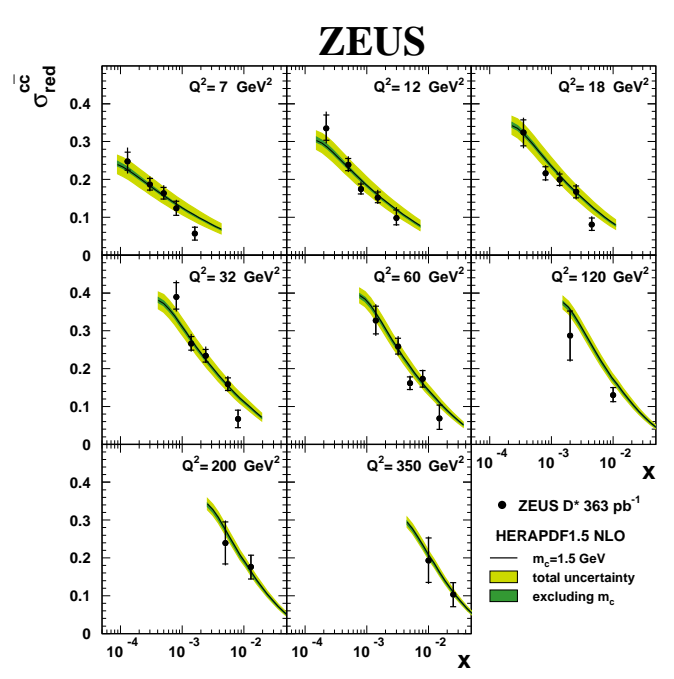

Figure 15: $\sigma^{c \bar{c}}$ determined from the ZEUS $D^{*}$ data compared to predictions from HERAPDF1.5 with $m_{c}=1.5 \mathrm{GeV}$.

\section{Charm fragmentation functions}

Charm fragmentation functions describe the probabilities for charm quarks to decay to specific charmed particles.

$$
f(c \rightarrow \text { charm particle })=\frac{\sigma(\text { charm particle })}{\sigma(\text { all charm particles })}
$$

Charm fragmentation functions are universal, i.e. independent of the production process of charm quarks. ZEUS measured the production of $D^{0}, D^{+}, D^{*+}, D_{s}, \Lambda_{c}^{+}$in photproduction events, i.e. $Q^{2} \leq$ $1 \mathrm{GeV}^{2}$, to determine the fragmentation functions [10]. Charge conjugate states are included. As examples, the signals for $D_{s}$ and $\Lambda_{c}^{+}$are shown in figures 16 and 17. The $D_{s}^{+}$mesons have been identified by the decay chain $D_{s}^{+} \rightarrow \Phi \pi^{+}$followed by $\Phi \rightarrow K^{+} K^{-}$. The lower peak at $1860 \mathrm{MeV}$ in figure 16 originates from the decay $D^{+} \rightarrow K^{+} K^{-} \pi^{+}$. The $\Lambda_{c}^{+}$baryons have been detected through their decay $\Lambda_{c}^{+} \rightarrow K^{-} p \pi^{+}$. Background from $D^{+}, D_{s}^{+} \rightarrow 3$ charged particle reflections has been subtracted according to a Monte Carlo model.

Figure 18 shows the determined charm fragmentation functions.

The values labeled ZEUS HERAII are the recent data. Corrections for cross-sections of not observed states $\left(\Xi_{c}^{+}, \Xi_{c}^{-}, \Omega_{c}^{0}\right)$ have been applied using PYTHIA MC calculations. This results in a correction factor of 1.14. They are compared with older measurements from the HERAI running 


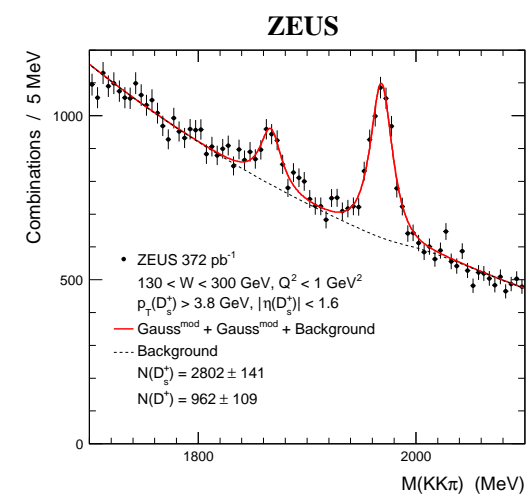

Figure 16: Inclusive $D_{s}^{+}$signal as measured in photoproduction events. The $D_{s}^{+}$mesons have been identified by the decay chain $D_{s}^{+} \rightarrow \Phi \pi^{+}$ followed by $\Phi \rightarrow K^{+} K^{-}$. The lower peak at $1860 \mathrm{MeV}$ originates from from the decay $D^{+} \rightarrow$ $K^{+} K^{-} \pi^{+}$.

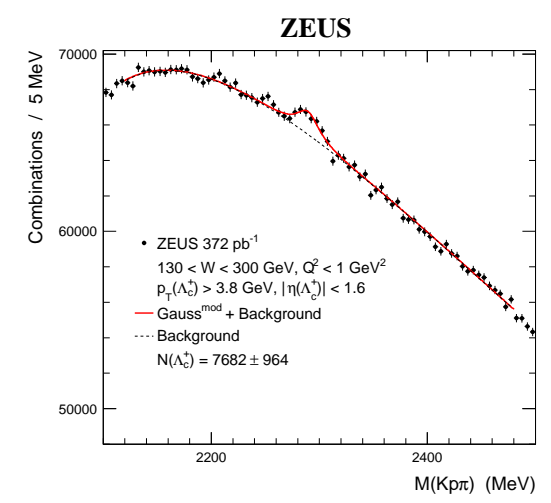

Figure 17: Inclusive $\Lambda_{c}^{+}$signal as measure in photoproduction events. The $\Lambda_{c}^{+}$ baryons have been detected through their decay $\Lambda_{c}^{+} \rightarrow K^{-} p \pi^{+}$. Background from $D^{+}, D_{s}^{+} \rightarrow$ 3 charged particle reflections is subtracted according to a Monte Carlo model.

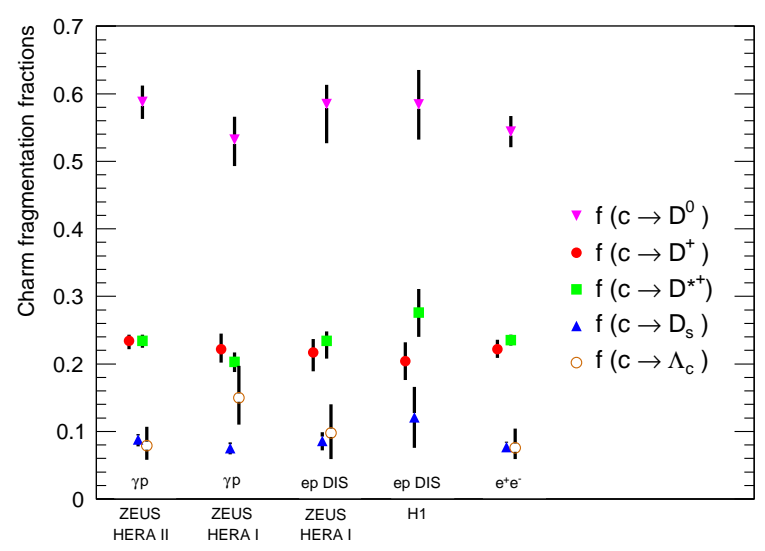

Figure 18: Charm fragmentation functions from HERAI and HERAII datasets and from $e^{+} e^{-}$annihilation.

period from ZEUS, labeled ZEUS HERAI, for photoproduction $(\gamma p)$ and for deep inelastic scattering events (ep DIS) from ZEUS [11],[12],[13] and H1 [14] as well as fragmentation functions measured in $e^{+} e^{-}-$scattering processes [15],[16]. There is agreement between all datasets and the data show that the charm fragmentation functions are universal. From the recent ZEUS data the fraction of charged $D$ mesons produced in a vector state has been determined to be

$$
\left.P_{V}^{D}=0.595 \pm 0.020(\text { stat. }) \pm 0.015 \text { (syst. }\right) \pm 0.011(B R) .
$$

From simple spin counting one expects a value 0.75 . The LUND string fragmentation model gives 0.66. Another important quantity is the strangeness suppression in charm production, defind by $\gamma_{s}=2 \sigma\left(D_{s}+\right) /\left[\sigma\left(D^{+}\right)+\sigma\left(D^{0}\right)\right]$. The measured value from the recent ZEUS data is

$$
\gamma_{s}=0.214 \pm 0.013(\text { stat. })_{-0.017}^{+0.006}(\text { syst. }) \pm 0.012(B R) .
$$


This has to be compared to non-charm strange particle production which gives $0.22-0.30$.

\section{Inelastic $J / \Psi$ and $\Psi^{\prime}$ photoproduction}

The inclusive production of $J / \Psi$ mesons in ep scattering can proceed via two processes: a colour singlet contribution and a colour octet contribution. This is shown in figures 19 a,b.

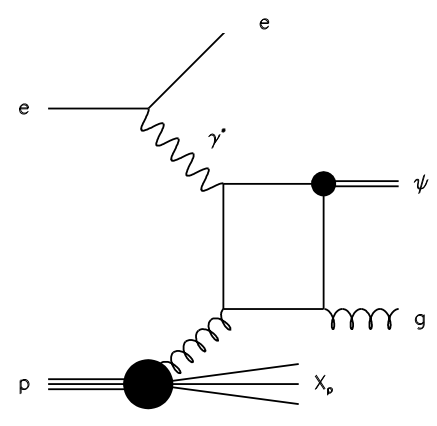

(a)

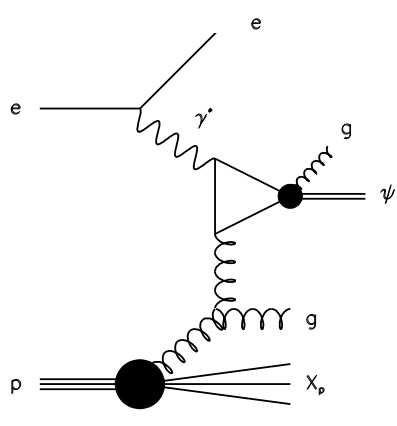

(b)

Figure 19: (a): Diagram for the colour singlet contribution (CS) to inclusive $J / \Psi$ production. (b): Diagram for the colour octet (CO) contribution.

New ZEUS measurements have been made for inclusive $J / \Psi$ and $\Psi^{\prime}$ photoproduction [17], detecting the mesons by their decays into muon pairs: $J / \Psi \rightarrow \mu^{+} \mu^{-}$and $\Psi^{\prime} \rightarrow \mu^{+} \mu^{-}$. Figure 20 shows their signals.

\section{ZEUS}
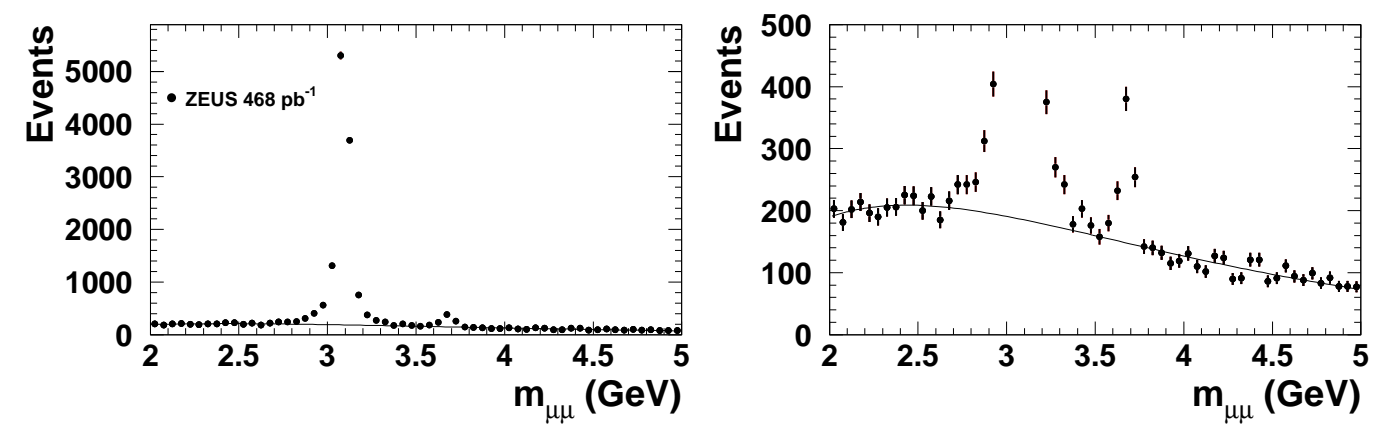

Figure 20: Mass spectrum of muon pairs showing the $J / \Psi$ and $\Psi^{\prime}$ signals. The right plot shows the enhanced region of the $\Psi^{\prime}$ signal.

It is a long standing question in $J / \Psi$ production whether an octet contribution really contributes. One indication might be the ratio between $\Psi^{\prime}$ and $J / \Psi$ production. This is presented in figure 21 as a function of $\mathrm{W}$, the photon-proton center of mass energy, the transverse momentum of the $J / \Psi$, $p_{T}$, and the the fraction of the photon energy transferred to the $J / \Psi, z=\left(E-p_{z}\right)_{\Psi} /\left(E-p_{z}\right)$ where $P_{z}$ is the momentum along the z-axis of the $J / \Psi$ and for all final state particles respectively. 
ZEUS
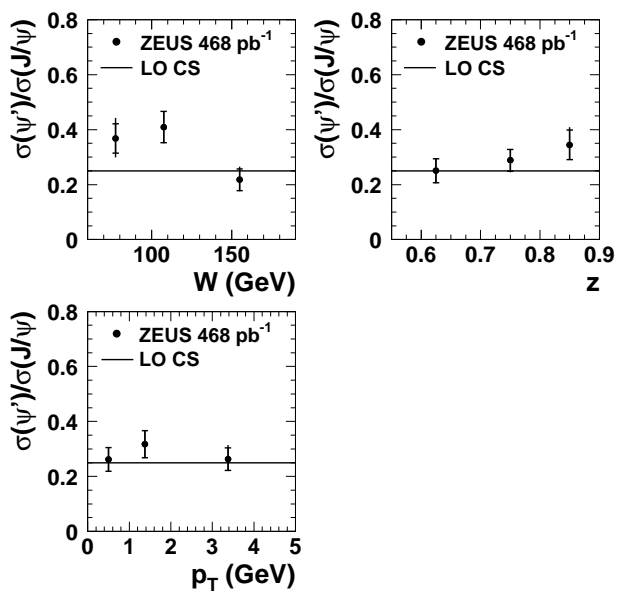

Figure 21: Ratios of $\Psi^{\prime}$ to $J / \Psi$ production as functions of $\mathrm{W}, P_{T}$, and $\mathrm{z}$. The ratios are compared to a lowest order calculation with only a colour singlet contribution, LO CS.

There is reasonable agreement between the data and the results from a leading order calculation with only a colour singlet contribution, LO CS.

Figure 22 presents the cross section $\frac{d \sigma(\gamma p \rightarrow J / \Psi X)}{d p_{T}^{2}}$ as a function of $p_{T}^{2}$ for several bins in z. The data are compared to NLO CS and NLO CS+CO calculations [18],[19]. It appears that the colour singlet contribution alone cannot describe the data sufficiently.

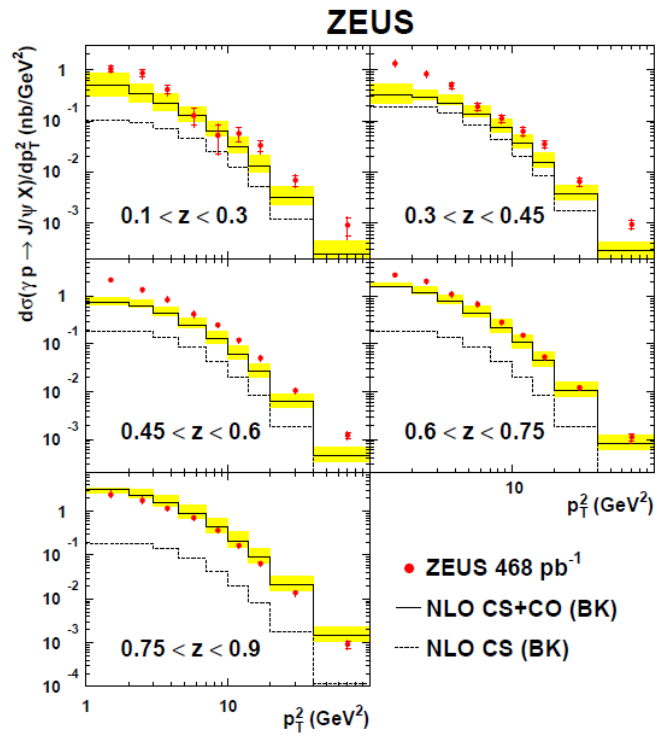

Figure 22: The differential cross-section $\frac{d \sigma(\gamma p \rightarrow J / \Psi X)}{d p_{T}^{2}}$ as a function of $p_{T}^{2}$ for several bins in z. Data are compared to NLO CS and NLO CS+CO calculations. 


\section{Prompt photon plus jet in photoproduction}

Prompt photons in DIS are photons which do not appear as decay products of final state particles but which originate directly from the hard scattering process as explained in figure 23 . This should not be confused with distinguishing photoproduction processes where the exchanged photon participates either directly in the hard scattering, which is called direct photoproduction, or the exchanged photon first turns into a hadronic state out of which a photon enters the hard scattering, which is called resolved photoproduction.
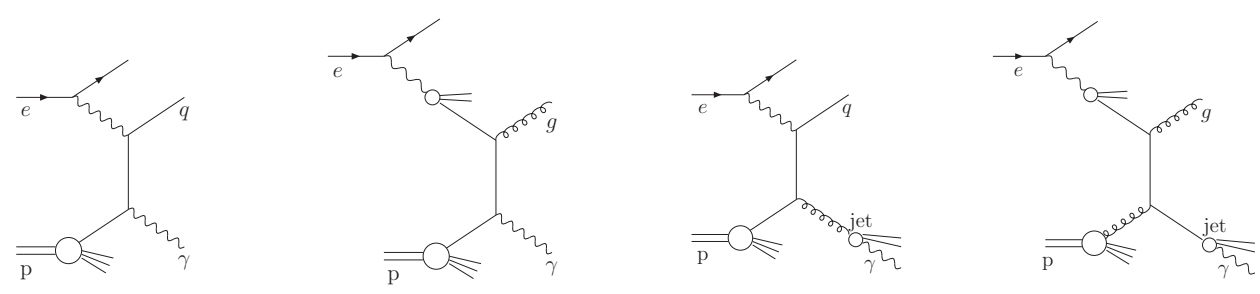

Figure 23: Examples of direct-prompt (first diagram) and resolved-promt (second diagram) processes at leading order, and photons from fragmantation processes for direct (third diagram) and resolved (fourth diagram) production processes.

ZEUS used photoproduction data with an identified jet in the final state to select prompt photons [20]. The selection was done with the help of the shower shape of isolated photons in the electromagnetic calorimeter:

$$
<\delta Z>=\frac{\sum_{i} E_{i}\left|Z_{i}-Z_{\text {cluster }}\right|}{w_{\text {cell }} \sum_{i} E_{i}} .
$$

$Z$ is the coordinate along the surface of the calorimeter, $i$ is the index of a calorimeter cell belonging to an identified cluster, $Z_{i}$ its position, $E_{i}$ its energy content and $Z_{\text {cluster }}$ is the center of gravity of the cluster. All electromagnetic calorimeter cells have the same width $w_{\text {cell }}$.

Figure 25 shows the energy weighted width $\langle\delta Z>$ of electromagnetic clusters for events with a jet and a photon. The main background to prompt photons comes from $\pi^{0} \rightarrow \gamma \gamma$ decays where both photons appear under a very small angle. These decays show up as a peak in the $\langle\delta Z\rangle$ destribution around 0.55 . The prompt photons form the peak at about 0.1 . The prompt photon signal has been extracted by a template fit to the $\langle\delta Z\rangle$ destribution.

The fraction of the energy of the exchanged photon which takes part in in the hard scattering is one for direct photoproduction processes and less than one for resolved photoproduction. Experimentally, this ratio is measured as:

$$
x_{\gamma}^{\text {meas }}=\frac{E^{\gamma}+E^{j e t}-p_{Z}^{\gamma}-p_{z}^{j e t}}{E^{\text {event }}-p_{Z}^{\text {event }}} .
$$

Figure 24 shows three differential cross-sections for prompt photon plus jet data and compares them to LO and NLO calculations. 


\section{ZEUS}

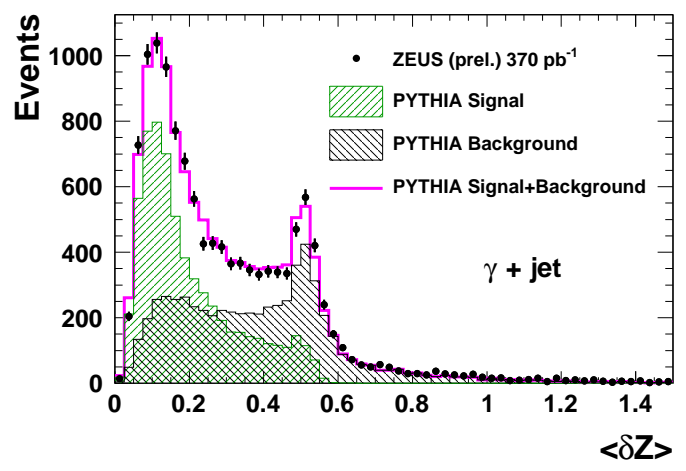

Figure 24: Energy weighted width of photon clusters in the calorimeter. For details see text.

\section{ZEUS}

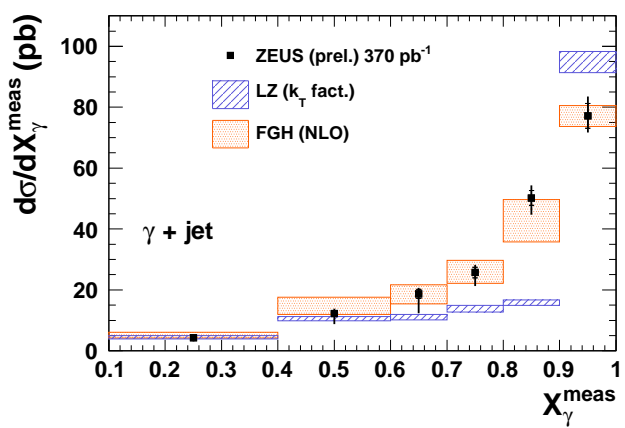

ZEUS

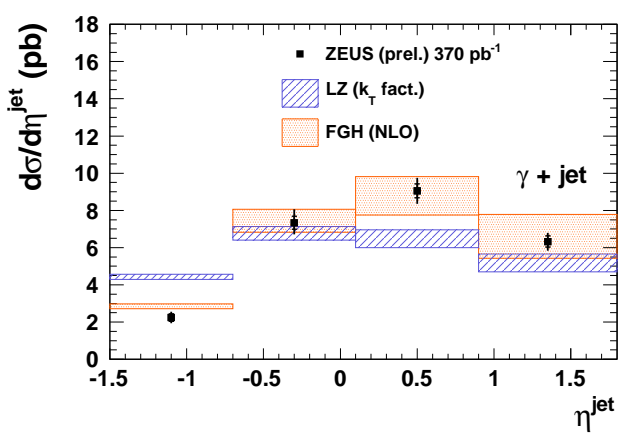

\section{ZEUS}

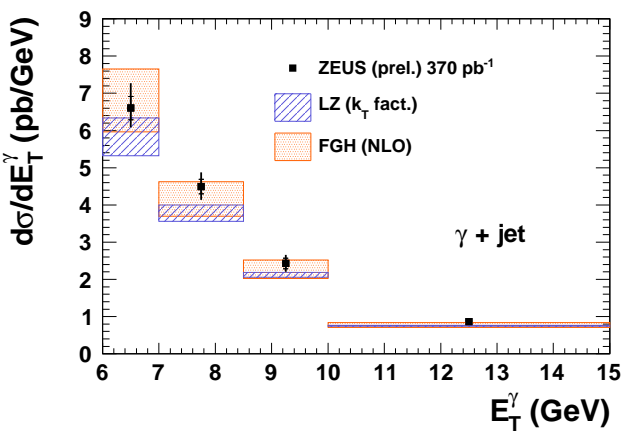

The NLO calculation (FHG) [21] provides a good description of the data given the uncertainties of the calculations and the measurements. The LO calculation (LZ) [22] describes the measurements reasonably well. However, an improved calculation is being prepared currently.

Figure 25: Differential cross-sections for prompt photon plus jet photoproduction.

\section{Summary}

The combination of charm results from $\mathrm{H} 1$ and ZEUS increased considerably the precision of charm cross-section measurements. It provides further constraints for the proton structure function when included in the dataset for PDF fits. New data for $D^{ \pm}$and $D^{*}$ are available. Work is underway to include them in the $\mathrm{H} 1$ and Zeus combination of charm data. The choice of an optimal charm mass for various schemes of heavy flavours in PQCD calculations reduces the differences in predictions between the different schemes. The measurement of charm fragmentation functions in 
photoproduction is in agreement with results from $e^{+} e^{-}$-scattering and is of comparable precision. New measurements of $J / \Psi$ and $\Psi^{\prime}$ photoproduction give indications for the need of colour octet contributions. The updated analysis of prompt photon production together with a jet provides an improved check of $\mathrm{pQCD}$ calculations without the complication of hadronisation corrections.

\section{References}

[1] H1 and ZEUS Collaborations, F.D.Aaron et al., Eur. Phys. J. C73 (2013) 2311.

[2] H1 and ZEUS collaborations, "HERAPDF1.5", LHAPDF grid available on: http ://www.desy.de/h1zeus/combined_results/index.php?do = proton_structure ;

V. Radescu, Proceedings of the $35^{\text {th }}$ International Conference of High Energy Physics, Proceedings of Science, POS (ICHEP 2010) 168.

[3] R.S. Thorne, Phys. Rev. D 73, (2006) 054019 [hep-ph/0601245].

[4] R.S. Thorne, arXiv:1201.6180.

[5] H.L. Lai et al., Phys.Rev. D 82, (2010) 074024 [arXiv:1007.2241].

[6] M.A.G. Aivazis, F.I. Olness and W.-K. Tung, Phys. Rev. D 50, (1994) 3085 [hep-ph/9312318];

M.A.G. Aivazis et al., Phys.Rev. D 50, (1994) 3102 [hep-ph/9312319].

[7] ZEUS Collaboration; H. Abramowicz et al., JHEP 05 (2013) 023; DESY-13-028.

[8] B.W. Harris and J. Smith, Phys.Rev. D 57, 2806 (1998).

[9] ZEUS Collaboration, H. Abramowicz et al., JHEP 05 (2013) 097; DESY-13-028.

[10] ZEUS Collaboration, H. Abramowicz et al., JHEP 09 (2013) 058; DESY-13-106.

[11] ZEUS Collaboration, S. Chekanov et al., Eur. Phys. J. C 44,351 (2005).

[12] ZEUS Collaboration, S. Chekanov et al., JHEP 07, 074 (2007).

[13] ZEUS Collaboration, H. Abramowicz et al., JHEP 11, 1 (2010).

[14] H1 Collaboration, A. Aktas et al., Eur. Phys. J. C 38, 447 (2004).

[15] L. Gladilin, hep-ex/9912064, 1999.

[16] E. Lohrmann hep-ex/11123757, 2011.

[17] ZEUS Collaboration, H. Abramowicz et al., JHEP 02 (2013) 071; DESY-12-226.

[18] T. Sjöstrand et. al., Comp. Phys. Comm. 135, 228 (2001);

E. Norrbin and T. Sjöstrand, Eur. Phys J. C 17, 137 (2000);

T. Sjöstrand, L. Lönnblad and S. Mrenna, hep-ph/0108264 (2001).

[19] M. Butenschön and B.A. Kniehl, Phys Rev. Lett. 104, 072001 (2010);

M. Butenschön and B.A. Kniehl, Phys Rev. D 84, 051501(R) (2011).

[20] ZEUS-prel-13-001.

[21] M. Fontannaz, J.Ph. Guillet and G. Heinrich, Eur. Phys. J. C 21 (2001) 303.

[22] A.V. Lipatov, M.A. Malyshev and N.P. Zotov, arXiv:1307.3644 (2013). 